\title{
BMJ Open The relationship between smoking status and health-related quality of life among smokers who participated in a 1-year smoking cessation programme in Taiwan: a cohort study using the EQ-5D
}

\author{
Pei-Ching Chen, ${ }^{1,2}$ Raymond Nien-Chen Kuo, ${ }^{3}$ Chih-Kuan Lai, ${ }^{4}$ Shih-Tzu Tsai, ${ }^{5,6}$ \\ Yue-Chune Lee ${ }^{1}$
}

To cite: Chen P-C, Kuo RN-C, Lai C-K, et al. The

relationship between smoking status and health-related quality of life among smokers who participated in a 1-year smoking cessation programme in Taiwan: a cohort study using the EQ-5D. BMJ Open 2015;5:e007249. doi:10.1136/ bmjopen-2014-007249

- Prepublication history for this paper is available online. To view these files please visit the journal online (http://dx.doi.org/10.1136/ bmjopen-2014-007249).

Received 23 November 2014 Revised 30 March 2015 Accepted 9 April 2015

CrossMark

For numbered affiliations see end of article.

Correspondence to Dr Yue-Chune Lee; yclee@ym.edu.tw

\section{ABSTRACT}

Objective: To assess the relationship between smoking status and health-related quality of life 1 year after participation in a smoking cessation programme in Taiwan.

Design: A cohort study of smokers who voluntarily participated in a smoking cessation programme with two follow-up assessments of smoking status via telephone interview, conducted 6 months and 1 year after finishing the smoking cessation programme.

Setting: Hospitals and clinics providing smoking cessation services.

Participants: A total of 3514 participants completed both telephone interviews, which represents a response rate of $64 \%$. After the interviews, participants were divided into four groups according to their smoking status: (1) long-term quitters: participants who had quit tobacco use for 1 year; (2) short-term quitters: participants who had been smoking for at least 6 months and then quit tobacco for 6 months after participating in the programme; (3) relapsed smokers: participants who relapsed into tobacco use after ceasing tobacco use for 6 months; and (4) continuing smokers: participants who failed to quit smoking for at least 1 year, despite participating in the programme.

Interventions: The Outpatient Smoking Cessation Service of Taiwan provides counselling and pharmacotherapy to individuals seeking to quit smoking. Primary outcomes: The health-related quality of life of the participants was measured using an approved Chinese version of the EuroQol-5D-3L (EQ-5D-3L) descriptive system.

Results: After controlling for sex, age, education, marital status, job status, monthly income and disease status at baseline, our results revealed that long-term ( $\mathrm{OR}=0.61$ (0.48 to 0.77$))$ and short-term (OR=0.65 (0.54 to 0.79$)$ ) quitters experienced less anxiety and depression than did continuing smokers.

Conclusions: Our study provides evidence to support claims that all quitters, regardless of whether they stop smoking for 6 months or 1 year, have better quality of life with regard to anxiety or depression.
Strengths and limitations of this study

- Unlike previous studies that used health-related quality of life to study quitters and smokers, this study assessed differences among quitters, relapsed smokers and continuing smokers, with regard to health-related quality of care using the EuroQol-5D (EQ-5D).

- This was a longitudinal study based on two telephone surveys conducted 6 months and 1 year after the completion of an outpatient smoking cessation programme. The generalised estimation equation method was used for repeated measures.

- The fact that smokers in this study volunteered to quit smoking means that self-selection bias was inevitable. In addition, smoking status was self-reported, such that the validity of the responses cannot be guaranteed.

- This study did not collect EQ-5D data at baseline (prior to quitting smoking), which made it impossible to control for differences between groups.

- The response rate of this study was $64 \%$, as this was the percentage of participants who were able to complete both follow-up surveys. Effects from the loss of participants are unknown.

\section{INTRODUCTION}

The adverse effects of smoking and the beneficial effects of smoking cessation have been well established. ${ }^{2}$ The harmful effects of smoking on health are well known; however, the influence of smoking cessation on health-related quality of life (HRQOL) over time has yet to be fully elucidated. HRQOL is broadly defined through the examination of several components of day-to-day functioning and well-being, such as physical functioning; functioning in daily activities, such as work and social activities; and psychological 
distress and well-being. ${ }^{3}$ A number of cross-sectional studies have focused on the differences in HRQOL among smokers, non-smokers and former smokers. ${ }^{4-7}$ These studies have shown that smokers tend to have poorer physical and mental health, compared with nonsmokers and former smokers, particularly with regard to depressive symptoms.

Previous longitudinal studies have focused on the relationship between smoking cessation and changes in HRQOL. ${ }^{8-14}$ However, two of these studies were limited with regard to follow-up duration ( $<6$ months), ${ }^{11} 14$ while others targeted specific groups such as females ${ }^{12}$ or university graduates, ${ }^{13}$ and still others included small samples. ${ }^{10}{ }^{11}{ }^{14}$ Hays et at compared various forms of pharmacotherapy used to assist in the cessation of smoking and the influence of these pharmacotherapies on HRQOL. Only two studies have compared the changes in HRQOL that occur among smokers and quitters. $^{8}{ }^{12}$ Piper et $a l^{8}$ used data obtained from smokers enrolled in a long-term smoking cessation trial and tracked changes in HRQOL over a period of 3 years. They found that, compared with smokers, quitters had improved global QOL and HRQOL at the end of the first and third years. Sarna et al $l^{12}$ evaluated the impact of quitting smoking on changes in HRQOL over an 8-year period among women in two cohorts. Continuing smokers and those who had quit smoking both presented a significant decline in short-term Form-36 (SF-36) physical component scores over time and significant improvements in SF-36 mental component scores at 8 years.

Various hypotheses have been proposed to explain the relationship between smoking and mental health: (1) smoking and poor mental health may share common causes (genetic factors or environmental mechanisms); (2) among individuals with poor mental health, smoking may be a coping strategy used to regulate psychiatric symptoms; and (3) smoking worsens mental health. ${ }^{15}$ Taylor et $a l^{16}$ reviewed 26 longitudinal studies and found that smoking cessation is associated with reduced depression, anxiety and stress, as well as improved mood and quality of life, compared with individuals who continue smoking. However, little is known about the changes in HRQOL that occur in relapsed smokers, compared with quitters and smokers.

The Outpatient Smoking Cessation Service (OSCS) of Taiwan was launched in 2002 to provide counselling and pharmacotherapy for individuals attempting to quit smoking. Doctors received an additional stipend for the delivery of cessation counselling and medications were subsidised for participants. Smokers who participated in the programme received treatment over an 8-week period. Beginning in 2005, the government increased reimbursements and medication subsidies in order to promote participation; however, due to a budget shortage, funding was reduced in April 2006. Previous studies have explored the effects of the OSCS programme with regard to provider participation and patient utilisation, ${ }^{17}$ the number of patients receiving counselling after the cutback in reimbursements, ${ }^{18}$ abstinence rates $^{19}$ and cost-benefit analysis. ${ }^{20}$ However, few researchers have compared the HRQOL among smokers, relapsed smokers and quitters, over a given duration. The aim of this study was to use EuroQol-5D (EQ-5D) as a means to assess differences in HRQOL among quitters, relapsed smokers and non-quitters who had participated in the OSCS in Taiwan.

\section{MATERIALS AND METHODS}

This cohort study had a study population of smokers who participated in the OSCS programme between January and September 2007. Data related to individual cases were provided by hospitals or clinics in monthly reports to the Smoking Cessation Therapy Management Center of Health Promotion Administration. A total of 115945 participants were enrolled in the OSCS between January and September 2007, approximately $14 \%$ of whom (2000 cases per month) were selected using systematic sampling for follow-up observation of their smoking status via telephone interviews. The OSCS programme was continuously open for enrolment, with new recruits joining each month throughout the study period. As a result, the telephone surveys were conducted on an ongoing basis. A total of 16274 individuals participated in the OSCS programme was sampled for the survey. Excluding ineligible cases left 12116 cases to be contacted. Among these cases, 5965 could not be contacted and 650 refused to be interviewed. A total of 5501 participants successfully completed the first interview. For more detailed information, refer to the previous study. ${ }^{20}$

The initial telephone survey of 5501 participants was conducted 6 months after the participants participated in the OSCS programme (between July 2007 and March 2008). A second telephone survey of 3514 participants from the first survey group was conducted 6 months after the first interview (between January 2008 and September 2008). This represents a response rate of $64 \%$. Researchers attempted to contact each of the participants three times by telephone for follow-up. From the 5501 cases in the initial survey, 281 were deemed lost due to having an invalid phone number or had moved away; 1544 cases were not at home, did not answer the phone, or the line was busy; and 162 cases refused to participate in the interview. A total of 3514 participants completed both interviews. The first interview was used to collect information related to demographic characteristics, current smoking status and current EQ-5D. A second interview 6 months later was used to collect information related to smoking status and current EQ-5D (1 year postprogramme).

The smoking status of participants was self-reported and determined by having the participants answer the following question in both of the follow-up interviews: "In the last 6 months, how many days have you abstained 
from cigarette use? (1) Less than 1 day, (2) 1 to 6 days (less than one week), (3) 7-29 days (one week to less than a month), (4) 30-179 days (1 month to less than 6 months), (5) 180 days (6 months or more)." Respondents who reported having quit for 180 days were considered quitters, while the others were considered smokers. According to answers from the two phone interviews, we had four conditions: (1) cases in which both answers indicated 180 days of abstinence were classified as long-term quitters; (2) cases where both answers indicated smoking for 180 days were classified as continuing smokers; (3) cases where the first answer was abstinence for 180 days with a second answer of smoking were classified as relapsed smokers; and (4) cases where the first answer indicated continued smoking for 180 days with a second answer of having quit for at least 180 days were classified as short-term smokers. Thus, participants were divided into four groups according to their smoking status: (1) long-term quitters: participants who had quit tobacco use for 1 year; (2) short-term quitters: participants who had been smoking for 6 months and then quit tobacco for 6 months after participating in the programme; (3) relapsed smokers: participants who had relapsed into tobacco use after 6 months cessation; and (4) continuing smokers: participants who failed to quit smoking for at least 1 year, despite participating in the programme.

This study used a standardised instrument "EQ-5D" for the measurement of generic HRQOL, in order to obtain a simple descriptive profile based on five dimensions applicable to a wide range of health conditions and treatments. ${ }^{21}$ The EQ-5D has been widely used in numerous countries in a variety of research fields. ${ }^{22}$ The EQ-5D comprises five dimensions: mobility, self-care, usual activities, pain/discomfort and anxiety/depression as well as a visual analogue scale (VAS) for health status. The EQ-VAS records the respondent's self-rated health on a vertical, VAS where the end points are labelled ' 100 ' (best imaginable health state) and ' 0 ' (worst imaginable health state). We used an approved Chinese version of the EQ-5D-3L with three levels of severity for each dimension: no health problems, slight health problems and extreme health problems. If no problems were reported for a given dimension, it was marked as level 1 , whereas extreme difficulty was marked as level 3. Because the EQ-5D is simple and short, it is ideal for telephone surveys.

The background characteristics of participants included sex, age, education, marital status, job status, monthly income and disease status at the first follow-up interview. The education of patients was categorised into three levels: junior high school or lower, senior high school and university/college or above. Marital status was classified as married, single and other (divorced, separated or widowed). Monthly income was separated into three levels: low $(<\mathrm{NT} \$ 20000 /$ month $)$, medium (NT\$20 001-NT\$49 999/month) and high ( $\geq$ NT $\$ 50000 /$ month). Disease status was determined by asking people about the diseases they have, such as cancers, cardiovascular diseases, heart or cerebrovascular disease, asthma, diabetes, chronic obstructive pulmonary disease, gastritis, nephritis and hepatitis. It should be noted that disease status did not include consideration of mental health condition.

\section{Missing data imputation}

A number of the covariates in the study were missing or unknown. In order to increase the number of samples, this study applied multiple regression imputation to fill in missing values related to monthly income, marital status and job status, which were missing in $1.6 \%, 0.65 \%$ and $0.51 \%$ of the cases, respectively. Imputation of monthly income was based on sex, education and job variables. Imputation of marital status was based on sex, age and education variables. Imputation of job status was based on sex, age and education variables.

\section{Sensitivity analysis}

Considering the fact that 'continuing smokers' included smokers who quit for less than 1 day as well as those who quit for 1-179 days, this group cannot be considered homogenous. We therefore conducted sensitivity subanalysis to differentiate between smokers who quit for less than 1 day and those who quit for 1-179 days in order to provide a stricter reclassification of the smoking group. Thus, the newly defined groups were as follows: (1) cases where both answers indicated 180 days of abstinence were classified as long-term quitters $(\mathrm{n}=262)$; (2) cases where both answers indicated abstinence for less than 1 day were classified as continuing smokers $(n=2271)$; (3) relapsed smokers: cases where the first answer was abstinence for 180 days and the second was abstinence for less than 1 day $(n=26)$; and (4) short-term smokers: cases where the first answer was abstinence for less than 1 day and the second answer indicated abstinence for 180 days $(n=96)$. The new classification system (as shown in table 1) resulted in a loss of 859 participants, which represents $24 \%$ of the cases in this study.

\section{Analysis}

The $\chi^{2}$ test and multinomial logistic regression were used to compare the proportions of demographic characteristics among the four groups. We dichotomised the EQ-5D levels into no problems (ie, level 1 as $\mathrm{Y}=0$ ) and problems (ie, levels 2 and 3 as $\mathrm{Y}=1$ ) due to the small number of responses citing level 3. Based on the distribution of the dependent variables, logistic regression was used for binary outcome variables and normal regression was used for VAS with the generalised estimation equation (GEE) method used for repeated measures. All statistical analysis was performed using SAS V.9.2 (SAS Institute, Cary, North Carolina, USA) with a $p$ value $<0.05$ considered significant. 
Table 1 New classification of smoking groups for sensitivity analysis

\begin{tabular}{|c|c|c|c|}
\hline & \multicolumn{3}{|c|}{ Number of days of abstinence from smoking during the first 6-month period } \\
\hline & Did not quit at all ( $<1$ day) & Quit 1-179 days & Quit completely ( $\geq 180$ days) \\
\hline \multicolumn{4}{|c|}{ Number of days of abstinence from smoking during the second 6-month period } \\
\hline Did not quit at all ( $<1$ day) & $\begin{array}{l}\text { Continuing smokers(C) } \\
n=2271\end{array}$ & $\begin{array}{l}x 1 \\
n=164\end{array}$ & $\begin{array}{l}\text { Relapsed quitters(R) } \\
n=26\end{array}$ \\
\hline Quit $1-179$ days & $\begin{array}{l}X 2 \\
n=269\end{array}$ & $\begin{array}{l}X 3 \\
n=120\end{array}$ & $\begin{array}{l}X 4 \\
n=19\end{array}$ \\
\hline Quit completely ( $\geq 180$ days) & $\begin{array}{l}\text { Short-term quitters(S) } \\
n=96\end{array}$ & $\begin{array}{l}x 5 \\
n=287\end{array}$ & $\begin{array}{l}\text { Long-term quitter(L) } \\
\mathrm{n}=262\end{array}$ \\
\hline
\end{tabular}

\section{RESULTS}

Following 6 months of participation in OSCS, a telephone survey of 5501 participants was conducted as a follow-up to the programme. Six months later, 3514 participants completed a second telephone interview, which represents a response rate of $64 \%$. Significant differences $(p<0.05)$ in age, marital status and monthly income, were observed among the smokers who completed both interviews and those who did not participate in the second follow-up. Smokers who did not participate in the follow-up were slightly younger (age $<30$ : $19.07 \%$ vs $13.52 \%$ ), a greater number were single $(24.97 \%$ vs $20.51 \%)$ and a greater number earned a medium monthly income ( $38.31 \%$ vs $34.73 \%)$, compared with those who completed both interviews.
A total of 307 participants reported 180 days of abstinence in the first interview, which represents a 6-month quit rate of $5.58 \%$. Among those quitters, 262 cases reported 180 days of abstinence in the second interview, such that the 12-month quit rate was $4.76 \%$. With regard to smoking status: 262 participants were identified as long-term quitters, 383 as short-term quitters, 45 as relapsed smokers and 2824 as continuing smokers.

As is shown in table 2, the four groups differed with regard to gender, age, marital status, job status and monthly income. However, a majority of the participants were male, 30-44 years old, had a high school education or above, were married, were currently employed, earned an above average monthly income and were free from disease.

Table 2 Demographic characteristics ( $N=3514)$

\begin{tabular}{|c|c|c|c|c|}
\hline Demographics & $\begin{array}{l}\text { Long-term quitters } \\
\mathrm{N}=262\end{array}$ & $\begin{array}{l}\text { Short-term quitters } \\
\mathrm{N}=383\end{array}$ & $\begin{array}{l}\text { Relapsed smokers } \\
\mathrm{N}=45\end{array}$ & $\begin{array}{l}\text { Smokers } \\
\mathrm{N}=\mathbf{2 8 2 4} \\
\end{array}$ \\
\hline $\operatorname{Sex}^{*}$ & Per cent & Per cent & Per cent & Per cent \\
\hline Male & 87.79 & 79.90 & 84.44 & 85.73 \\
\hline Female & 12.21 & 20.10 & 15.56 & 14.27 \\
\hline \multicolumn{5}{|l|}{ Age } \\
\hline$<30$ & 8.78 & 13.05 & 8.89 & 14.09 \\
\hline $30-44$ & 37.79 & 31.07 & 17.78 & 38.49 \\
\hline $45-59$ & 28.63 & 29.24 & 44.44 & 30.95 \\
\hline$\geq 60$ & 24.81 & 26.63 & 28.89 & 16.47 \\
\hline \multicolumn{5}{|l|}{ Education } \\
\hline Below junior school & 34.10 & 34.46 & 40.00 & 34.64 \\
\hline High school & 35.63 & 33.94 & 31.11 & 37.41 \\
\hline University/college above & 30.27 & 31.59 & 28.89 & 27.95 \\
\hline \multicolumn{5}{|l|}{ Marital status $\ddagger$} \\
\hline Single & 11.45 & 16.45 & 15.56 & 21.82 \\
\hline Married & 81.68 & 74.67 & 73.33 & 68.79 \\
\hline Separated, widowed & 6.87 & 8.88 & 11.11 & 9.39 \\
\hline \multicolumn{5}{|l|}{ Employed $\ddagger$} \\
\hline Yes & 63.98 & 62.14 & 60.00 & 74.42 \\
\hline No & 36.02 & 37.86 & 40.00 & 25.58 \\
\hline \multicolumn{5}{|l|}{ Monthly income(NT\$)† } \\
\hline Low $(\leq 20000)$ & 29.01 & 34.73 & 46.67 & 28.61 \\
\hline Medium (20 001-49 999) & 31.68 & 35.77 & 24.44 & 35.98 \\
\hline High $(\geq 50000)$ & 39.31 & 29.50 & 28.89 & 35.41 \\
\hline \multicolumn{5}{|l|}{ Any disease at baseline } \\
\hline Yes & 31.30 & 36.81 & 40.00 & 33.55 \\
\hline No & 68.70 & 63.19 & 60.00 & 66.45 \\
\hline
\end{tabular}


Multinomial logistic regression was used to test for differences among the four groups with regard to sex, age, marital status, employment status and income status, using continuing smokers as a reference group. Compared with continuing smokers, males were significantly less likely to be the short-term quitters $(\mathrm{OR}=0.66$, $95 \% \mathrm{CI}=0.50$ to 0.87 ). Individuals above the age of 60 were significantly more likely to be short-term quitters or long-term quitters (short-term quitters $\mathrm{OR}=1.75,95 \%$ $\mathrm{CI}=1.21$ to 2.51; long-term quitters $\mathrm{OR}=2.42,95 \%$ $\mathrm{CI}=1.48$ to 3.96$)$. Married individuals were significantly more likely to be short-term quitters or long-term quitters (short-term quitters $\mathrm{OR}=1.44,95 \% \mathrm{CI}=1.08$ to 1.92 ; long-term quitters $\mathrm{OR}=2.26,95 \% \mathrm{CI}=1.53$ to 3.35 ). Individuals with a job were significantly less likely to be relapsed smokers, short-term quitters or long-term quitters (relapsed smokers $\mathrm{OR}=0.52,95 \% \mathrm{CI}=0.28$ to 0.94; short-term quitters $\mathrm{OR}=0.56,95 \% \mathrm{CI}=0.45$ to 0.71 ; long-term quitters $\mathrm{OR}=0.61,95 \% \mathrm{CI}=0.47$ to 0.80 ). Individuals with a medium monthly income were significantly less likely to be relapsed smokers (relapsed smokers $\mathrm{OR}=0.42,95 \% \mathrm{CI}=0.20$ to 0.87 ); however, individuals with high monthly income were significantly less likely to be short-term quitters (short-term quitters $\mathrm{OR}=0.69,95 \% \mathrm{CI}=0.53$ to 0.90$)$.

Table 3 presents the EQ-5D among the four groups after participating in OSCS for 6 months and 1 year. Level 1 refers to situations involving no problems, level 2 refers to slight problems and level 3 refers to extreme problems. Very few of the four groups reported extreme health problems related to mobility, self-care, usual activities, or pain and anxiety, after participating in OSCS for 6 months and 1 year. However, approximately $30 \%$ of the participants reported slight health problems related to pain and anxiety. Finally, the mean VAS among the four groups was approximately 70 .

Table 4 presents the GEE results for EQ-5D among the four groups. After controlling for confounders, short-term quitters appeared to have fewer problems with regard to usual activities and pain/discomfort, compared with continuing smokers. In addition, long-term quitters and short-term quitters reported fewer problems related to anxiety/depression. Moreover, long-term quitters, short-term quitters and relapsed smokers had VAS scores higher than those of continuing smokers.

Among the smoking status groups, no significant differences were observed with regard to mobility or selfcare. Relapsed smokers reported fewer problems related to mobility, pain/discomfort and anxiety/depression; however, those results were not significant.

Male participants reported less pain/discomfort and anxiety/depression than their female counterparts ( $\mathrm{OR}=0.78,0.83$, respectively). Older individuals reported a greater number of problems related to mobility, usual activities and pain/discomfort (OR=2.16-2.98, 3.80$4.50,1.44-1.63$, respectively). Individuals with a higher education reported fewer problems related to mobility, usual activities and pain/discomfort $(\mathrm{OR}=0.50,0.61-$ $0.70,0.72-0.81$, respectively). Separated/widowed individuals reported a greater number of problems related to mobility, self-care, usual activities, pain/discomfort and anxiety/depression $\quad(\mathrm{OR}=1.50,2.11,1.45,1.40,1.28$,

Table 3 Comparison of EQ-5D among the four groups after participating in OSCS for 6 months and 1 year

\begin{tabular}{|c|c|c|c|c|c|c|c|c|}
\hline & \multicolumn{2}{|c|}{ Long-term quitters } & \multicolumn{2}{|c|}{ Short-term quitters } & \multicolumn{2}{|c|}{ Relapsed smokers } & \multicolumn{2}{|l|}{ Smokers } \\
\hline & 6 Months & 1 Year & 6 Months & 1 Year & 6 Months & 1 Year & 6 Months & 1 Year \\
\hline \multicolumn{9}{|l|}{ Mobility (\%) } \\
\hline Level 1 & 96.18 & 94.27 & 95.29 & 94.78 & 93.33 & 95.56 & 95.57 & 95.01 \\
\hline Level 2 & 3.82 & 5.73 & 4.45 & 5.22 & 6.67 & 4.44 & 4.36 & 4.85 \\
\hline Level 3 & 0.00 & 0.00 & 0.26 & 0.00 & 0.00 & 0.00 & 0.07 & 0.14 \\
\hline \multicolumn{9}{|l|}{ Self-care (\%) } \\
\hline Level 1 & 98.09 & 98.47 & 98.96 & 98.96 & 97.78 & 97.78 & 99.19 & 99.15 \\
\hline Level 2 & 1.53 & 1.15 & 0.52 & 1.04 & 2.22 & 2.22 & 0.71 & 0.74 \\
\hline Level 3 & 0.38 & 0.38 & 0.52 & 0.00 & 0.00 & 0.00 & 0.11 & 0.11 \\
\hline \multicolumn{9}{|c|}{ Usual activities (\%) } \\
\hline Level 1 & 94.66 & 94.64 & 95.04 & 96.34 & 88.89 & 91.11 & 94.58 & 95.04 \\
\hline Level 2 & 3.82 & 4.60 & 4.70 & 3.14 & 11.11 & 8.89 & 4.89 & 4.25 \\
\hline Level 3 & 1.53 & 0.77 & 0.26 & 0.52 & 0.00 & 0.00 & 0.53 & 0.71 \\
\hline \multicolumn{9}{|c|}{ Pain/discomfort (\%) } \\
\hline Level 1 & 75.95 & 73.28 & 79.00 & 74.41 & 71.11 & 73.33 & 71.33 & 69.59 \\
\hline Level 2 & 22.52 & 25.95 & 19.16 & 23.76 & 28.89 & 24.44 & 25.94 & 28.50 \\
\hline Level 3 & 1.53 & 0.76 & 1.84 & 1.83 & 0.00 & 2.22 & 2.73 & 1.92 \\
\hline \multicolumn{9}{|c|}{ Anxiety/depression (\%) } \\
\hline Level 1 & 76.25 & 72.41 & 71.54 & 72.51 & 66.67 & 64.44 & 63.20 & 63.52 \\
\hline Level 2 & 22.22 & 25.29 & 26.11 & 26.18 & 28.89 & 28.89 & 32.54 & 32.65 \\
\hline Level 3 & 1.53 & 2.30 & 2.35 & 1.31 & 4.44 & 6.67 & 4.26 & 3.83 \\
\hline VAS (mean) & 79.20 & 75.87 & 76.38 & 75.85 & 76.98 & 71.49 & 69.37 & 70.26 \\
\hline
\end{tabular}

Level 1=no problems, level 2=slight problems, level $3=$ extreme problems.

OSCS, Outpatient Smoking Cessation Service; VAS, visual analogue scale. 
Table 4 Generalised estimation equation results for EQ-5D among the four groups

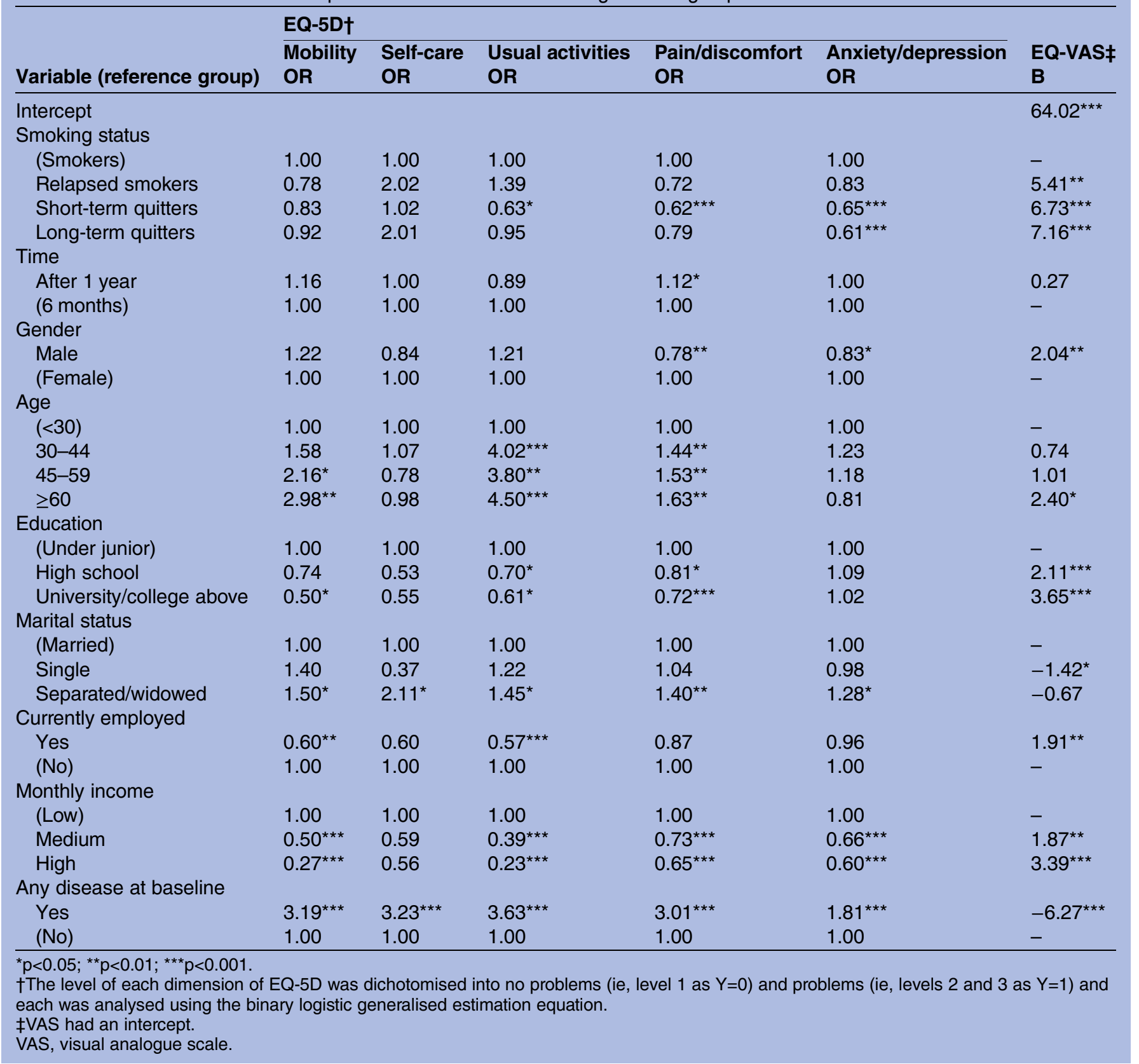

respectively). Employed individuals reported fewer problems related to mobility and usual activities $(\mathrm{OR}=0.60$, 0.57 , respectively). Individuals with medium or high monthly incomes reported fewer problems related to mobility $(\mathrm{OR}=0.50,0.27$, respectively), usual activities $(\mathrm{OR}=0.39,0.23$, respectively), pain/discomfort $(\mathrm{OR}=0.73$, 0.65 , respectively) and anxiety/depression $(\mathrm{OR}=0.66$, 0.60 , respectively). Individuals who noted a disease at baseline reported a greater number of problems related to mobility, self-care, usual activities, pain/discomfort and anxiety/depression $\quad(\mathrm{OR}=3.19,3.23,3.63,3.01,1.81$, respectively).

Males reported higher VAS scores than did their female counterparts $(B=2.04, p<0.001)$ and individuals older than 60 years reported higher VAS scores compared with those below 30 years of age $(B=2.40$, $\mathrm{p}<0.05)$. Individuals with a higher education reported higher VAS scores $(B=2.11-3.65, \mathrm{p}<0.001)$. Single individuals reported lower VAS scores than did married people $(\mathrm{B}=-1.42, \quad \mathrm{p}<0.05)$. Employed individuals reported higher VAS scores than did the unemployed $(\mathrm{B}=1.91, \mathrm{p}<0.01)$. Individuals with a higher monthly income reported higher VAS scores $(\mathrm{B}=1.87-3.39$, $\mathrm{p}<0.01)$. Individuals with disease reported lower VAS scores than did those who were free from disease $(B=-6.27$, $\mathrm{p}<0.001)$.

\section{Sensitivity analysis results}

The new classification of GEE results for EQ-5D among the four groups was shown in table 5. After controlling 
for confounders, short-term quitters appeared to have fewer problems with regard to pain/discomfort, compared with continuing smokers. After controlling for confounders, long-term quitters and short-term quitters reported fewer problems related to anxiety/depression. After controlling for confounders, long-term quitters and short-term quitters had VAS scores higher than those of continuing smokers. We found the results were similar to our original results in table 4 .

\section{DISCUSSION}

This study provides evidence that quitting smoking can benefit one's subjective assessment of anxiety or depression. In this study, this effect is particularly evident among individuals who quit for extended periods (>12 months) as well as those who quit for short durations (6 months), following participation in OSCS for 1 year. Our results revealed that quitting smoking, whether for a short or long period of time, had a significant effect on reducing anxiety or depression.

This is the first study to use EQ-5D to explore the relationship between smoking status and changes in HRQOL. We found long-term and short-term quitters both had fewer problems related to anxiety or depression. Our findings are similar to those obtained in previous longitudinal studies; however, we used different instruments, therefore, we cannot make a direct comparison with their results. In a review of previous studies,

Table 5 New classifications of generalised estimation equation results for $E Q-5 D$ among the four groups

\begin{tabular}{|c|c|c|c|c|c|c|}
\hline \multirow[b]{2}{*}{ Variable (reference group) } & \multicolumn{5}{|l|}{ EQ-5D† } & \multirow[b]{2}{*}{$\begin{array}{l}\text { EQ-VAS } \neq \\
\text { B }\end{array}$} \\
\hline & $\begin{array}{l}\text { Mobility } \\
\text { OR }\end{array}$ & $\begin{array}{l}\text { Self-care } \\
\text { OR }\end{array}$ & $\begin{array}{l}\text { Usual activities } \\
\text { OR }\end{array}$ & $\begin{array}{l}\text { Pain/discomfort } \\
\text { OR }\end{array}$ & $\begin{array}{l}\text { Anxiety/depression } \\
\text { OR }\end{array}$ & \\
\hline Intercept & & & & & & $61.93^{\star \star \star}$ \\
\hline \multicolumn{7}{|l|}{ Smoking status } \\
\hline (Smokers) & 1.00 & 1.00 & 1.00 & 1.00 & 1.00 & - \\
\hline Relapsed smokers & 0.76 & 3.42 & 1.54 & 0.99 & 0.87 & 3.49 \\
\hline Short-term quitters & 1.09 & 1.04 & 0.78 & $0.53^{\star \star}$ & $0.62^{\star \star}$ & $3.86^{\star \star}$ \\
\hline Long-term quitters & 1.02 & $2.69^{*}$ & 1.00 & 0.79 & $0.60^{\star \star \star}$ & $7.78^{\star \star \star}$ \\
\hline \multicolumn{7}{|l|}{ Time } \\
\hline After 1 year & 1.12 & 1.10 & 0.89 & $1.14^{*}$ & 1.00 & 0.41 \\
\hline (6 months) & 1.00 & 1.00 & 1.00 & 1.00 & 1.00 & - \\
\hline \multicolumn{7}{|l|}{ Gender } \\
\hline Male & 1.16 & 0.91 & 1.13 & $0.70^{\star \star}$ & $0.81^{*}$ & $2.45^{\star *}$ \\
\hline (Female) & 1.00 & 1.00 & 1.00 & 1.00 & 1.00 & - \\
\hline \multicolumn{7}{|l|}{ Age } \\
\hline$(<30)$ & 1.00 & 1.00 & 1.00 & 1.00 & 1.00 & - \\
\hline $30-44$ & 1.31 & 0.59 & $2.85^{\star \star}$ & $1.52^{\star \star}$ & 1.17 & 1.44 \\
\hline $45-59$ & 2.01 & 0.47 & $2.79^{\star}$ & $1.57^{\star \star}$ & 1.13 & $2.03^{\star}$ \\
\hline$\geq 60$ & 2.31 & 0.32 & $3.33^{\star \star}$ & $1.67^{\star \star}$ & 0.78 & $4.44^{\star \star \star}$ \\
\hline \multicolumn{7}{|l|}{ Education } \\
\hline (Under junior) & 1.00 & 1.00 & 1.00 & 1.00 & 1.00 & - \\
\hline High school & 0.77 & 0.49 & 0.70 & 0.84 & 1.08 & $2.87^{\star \star \star}$ \\
\hline University/college above & $0.58^{*}$ & 0.77 & 0.68 & $0.69^{\star \star \star}$ & 0.99 & $3.50^{\star \star \star}$ \\
\hline \multicolumn{7}{|l|}{ Marital status } \\
\hline (Married) & 1.00 & 1.00 & 1.00 & 1.00 & 1.00 & - \\
\hline Single & 1.36 & 0.13 & 1.12 & 1.09 & 0.98 & -0.59 \\
\hline Separated/widowed & $1.74^{\star \star}$ & $3.00^{\star \star}$ & $1.61^{*}$ & $1.55^{\star \star \star}$ & $1.31^{*}$ & -0.98 \\
\hline \multicolumn{7}{|l|}{ Currently employed } \\
\hline Yes & $0.65^{\star}$ & 0.62 & $0.68^{*}$ & 0.92 & 1.03 & $1.91^{*}$ \\
\hline (No) & 1.00 & 1.00 & 1.00 & 1.00 & 1.00 & - \\
\hline \multicolumn{7}{|l|}{ Monthly income } \\
\hline (Low) & 1.00 & 1.00 & 1.00 & 1.00 & 1.00 & - \\
\hline Medium & $0.48^{\star \star \star}$ & 0.39 & $0.35^{\star \star \star}$ & $0.79^{\star}$ & $0.64^{\star \star \star}$ & 1.52 \\
\hline High & $0.23^{\star \star \star}$ & 0.34 & $0.21^{\star \star *}$ & $0.68^{* *}$ & $0.62^{* * *}$ & $3.12^{\star \star \star}$ \\
\hline \multicolumn{7}{|l|}{ Any disease at baseline } \\
\hline Yes & $3.11^{\star \star \star}$ & $2.58^{*}$ & $3.52^{\star \star \star}$ & $3.06^{\star \star \star}$ & $1.85^{\star \star \star}$ & $-6.51^{\star * *}$ \\
\hline (No) & 1.00 & 1.00 & 1.00 & 1.00 & 1.00 & - \\
\hline
\end{tabular}


Taylor et $a l^{16}$ discovered that smoking cessation is associated with reduced depression, anxiety and stress, and improved mood and quality of life, compared with individuals who continue smoking. Using the SF-36, Guiterrez-Bedmar et $a l^{13}$ found that continuing smokers had worse scores than did non-smokers with regard to general health and mental health, whereas recent quitters showed improvements in mental health over those who continued smoking and those who took up smoking. Using the SF-36, Sarna et al ${ }^{12}$ found that continuing smokers as well as quitters showed significant improvements in mental component scores at 8 years. Sales $e t a l^{10}$ also used the SF-36, and found that summary scores of mental and physical components were higher among quitters than among non-quitters after ceasing smoking for 12 months.

Our results show that those who quit smoking for 6 months were less likely to have problems related to usual activities, pain or discomfort, or anxiety or depression, than smokers. These results are similar to those obtained in previous cross-sectional studies. ${ }^{4-6}$ Mody and Smith $^{4}$ found that current smokers were more likely to report poor mental health status and limitations in their usual activities, compared with non-smokers and ex-smokers. McClave et a $\bar{l}$ found that former smokers and never-smokers were less likely to report depressive symptoms than were non-quitters. Mulder et at obtained lower summary scores for the mental component among current smokers compared with never-smokers and ex-smokers.

In this study, long-term quitters, short-term quitters and relapsed smokers presented EQ-VAS scores higher than those of continuing smokers. These findings are similar to those in previous studies using different HRQOL instruments, such as the SF-36, which includes eight dimensions. The discussion related to general health in the SF-36 is similar to our study; therefore, we opted to limit the comparison to this. Guiterrez-Bedmar et $a l^{13}$ reported that continuing smokers had worse scores than recent quitters with regard to general health. Sarna et $a l^{12}$ found that continuing smokers as well as quitters present a significant decline in general health scores, whereas continuing smokers presented a decline in a wider range of scores than did quitters at 8 years. Sales $e t a l^{10}$ found that the general health component scores were higher among quitters than among non-quitters after ceasing smoking for 12 months.

In contrast, our results show that smoking cessation had no significant effect on EQ-5D with regard to mobility or self-care. Our results differ from those obtained in previous studies, ${ }^{10}{ }^{12}$ where quitters had higher SF-36 scores for the physical component than did smokers. These findings can be explained by differences in the smoking cessation programmes and the background characteristics of participants. The participants in this study participated in a free smoking cessation service in which counselling and pharmacotherapy were provided. In the study by Sarna et $a l^{12}$ participants were registered nurses and were undecided as to whether they had been affected by smoking cessation programmes over an 8 -year period. In the study by Sales $e t a l,{ }^{10}$ the cohort included only 60 patients, who were selfreferred to a smoking cessation programme at a public hospital.

In this study, the 6-month quit rate was $5.58 \%$ and the 12 -month quit rate was $4.76 \%$. Naranjo et $a l^{23}$ reported smoking cessation rates of $14.4 \%$ and $15.7 \%$ at 6 and 12 months, respectively, among patients with arthritis in a rheumatology clinic. Our results could not be compared directly with previous studies due to fundamental differences in the design of the smoking cessation programme and study population. Chang et $a l^{17}$ reported on smoking cessation outpatient services with quit rates of $25.2 \%$ and $21.3 \%$ in a 6 -month follow-up in 2004 and 2005, respectively, in Taiwan. These differences could be explained by the fact that the quit rate was not strictly defined; that is, quitting for either 7 days or 6 months were both included in the quit rate.

VAS scores represent a subjective self-evaluation of one's health, and individuals over the age of 60 reported higher VAS scores than did those below the age of 30 . Whynes $^{24}$ examined the correspondence between EQ-VAS scores and EQ-5D health states, and found that VAS scores are influenced by EQ-5D health state classification; the participant's perceived locus of control; and by his/her age, educational attainment, ethnic origin and smoking behaviour. Whynes ${ }^{25}$ also examined how the relationship between health state classification and VAS score varied with medical condition. They found that a given change between two EQ-5D-defined health states could produce different changes in VAS scores for different medical conditions and interventions. The difference between EQ-VAS scores and EQ-5D health status may be due to other factors, such as disease status or sociodemographic factors.

This study faced a number of limitations. First, smoking status was self-reported; therefore, validity cannot be guaranteed. Second, we were unable to collect EQ-5D data at baseline (before quitting smoking), which made it impossible to control for differences between groups; however, we attempted to control for confounders and obtained two measurements, at 6 months and 1 year after participation in OSCS. Third, due to a lack of funding, we were able to follow participants for only 1 year, which may be too short-term to observe differences in the quality of life among smokers, quitters and relapsed smokers. In the future, researchers should conduct a long-term cohort study to obtain information related to the quality of life among smokers, quitters and relapsed smokers. Fourth, the smokers in this study volunteered to quit smoking; therefore, selfselection bias was inevitable. Finally, only $64 \%$ of the 5501 participants successfully completed both follow-up surveys, and the effect from a loss of participants is unknown. 
Our findings provide evidence to support the claims that all quitters, regardless of whether they stop smoking for 6 months or 1 year, have better quality of life with regard to mental health. These findings are important for governmental organisations such as the Health Promotion Administration. These findings provide additional evidence to encourage smokers to quit smoking. Future researchers could extend the follow-up to better understand the long-term effects of smoking cessation on quality of life.

\author{
Author affiliations \\ ${ }^{1}$ Institute of Health and Welfare Policy, College of Medicine, National Yang- \\ Ming University, Taipei, Taiwan \\ 2Department of Health and Welfare, School of Metropolitan Governance, \\ University of Taipei, Taipei, Taiwan \\ ${ }^{3}$ Institute of Health Policy and Management, College of Public Health, National \\ Taiwan University, Taipei, Taiwan, R.O.C \\ ${ }^{4}$ Department of Family Medicine, Taipei Veterans General Hospital, Taipei, \\ Taiwan \\ ${ }^{5}$ Center for Preventive Services, Hualien Tzu Chi Hospital, Buddhist Tzu Chi \\ Medical Foundation, Hualien, Taiwan \\ ${ }^{6}$ College of Medicine, Tzu Chi University, Hualien, Taiwan
}

Acknowledgements The authors would like to thank Ying-Fu Liao and Ming-Ta Liu at the Outpatient Smoking Cessation Management Center for their assistance in data collection.

Contributors P-CC contributed to the study design, statistical analysis, interpretation and writing of the manuscript. RN-CK contributed to the interpretation and writing of the manuscript. Y-CL contributed through coordination of the study, interpretation and writing of the manuscript, and acting as corresponding author. S-TT and C-KL contributed to data acquisition and interpretation as well as the writing of the manuscript. All authors have read and approved the content of the manuscript.

Funding This study was supported by the Health Promotion Administration, Ministry of Health and Welfare, Taiwan (No. 95039-1)

Competing interests None declared.

Provenance and peer review Not commissioned; externally peer reviewed.

Data sharing statement No additional data are available.

Open Access This is an Open Access article distributed in accordance with the Creative Commons Attribution Non Commercial (CC BY-NC 4.0) license, which permits others to distribute, remix, adapt, build upon this work noncommercially, and license their derivative works on different terms, provided the original work is properly cited and the use is non-commercial. See: http:// creativecommons.org/licenses/by-nc/4.0/

\section{REFERENCES}

1. US Centers for Disease Control and Prevention. Health effects of cigarette smoking. Secondary health effects of cigarette smoking, 2013. http://www.cdc.gov/tobacco/data_statistics/fact_sheets/health effects/effects_cig_smoking/index.htm

2. US Centers for Disease Control and Prevention. Health benefits of cessation. Secondary health benefits of cessation, 2013. http://www. cdc.gov/tobacco/data_statistics/fact_sheets/cessation/quitting/index. $\mathrm{htm} \#$ benefits

3. Stewart AL, King AC, Killen JD, et al. Does smoking cessation improve health-related quality-of-life? Ann Behav Med 1995; 17:331-8.

4. Mody RR, Smith MJ. Smoking status and health-related quality of life: as findings from the 2001 Behavioral Risk Factor Surveillance System data. Am J Health Promot 2006;20:251-8.

5. McClave AK, Dube SR, Strine TW, et al. Associations between health-related quality of life and smoking status among a large sample of U.S. adults. Prev Med 2009;48:173-9.

6. Mulder I, Tijhuis M, Smit HA, et al. Smoking cessation and quality of life: the effect of amount of smoking and time since quitting. Prev Med 2001;33:653-60.

7. Castro MR, Matsuo T, Nunes SO. Clinical characteristics and quality of life of smokers at a referral center for smoking cessation. J Bras Pneumol 2010;36:67-74.

8. Piper ME, Kenford S, Fiore MC, et al. Smoking cessation and quality of life: changes in life satisfaction over 3 years following a quit attempt. Ann Behav Med 2012;43:262-70.

9. Hays JT, Croghan IT, Baker CL, et al. Changes in health-related quality of life with smoking cessation treatment. Eur J Public Health 2012;22:224-9.

10. Sales MP, Oliveira MI, Mattos IM, et al. The impact of smoking cessation on patient quality of life. J Bras Pneumol 2009;35:436-41.

11. Erickson SR, Thomas LA, Blitz SG, et al. Smoking cessation: a pilot study of the effects on health-related quality of life and perceived work performance one week into the attempt. Ann Pharmacother 2004;38:1805-10.

12. Sarna L, Bialous SA, Cooley ME, et al. Impact of smoking and smoking cessation on health-related quality of life in women in the Nurses' Health Study. Qual Life Res 2008;17:1217-27.

13. Guiterrez-Bedmar M, Segui-Gomez M, Gomez-Gracia E, et al. Smoking status, changes in smoking status and health-related quality of life: findings from the SUN ("Seguimiento Universidad de Navarra") cohort. Int J Environ Res Public Health 2009:6:310-20.

14. Zillich AJ, Ryan M, Adams A, et al. Effectiveness of a pharmacist-based smoking-cessation program and its impact on quality of life. Pharmacotherapy 2002;22:759-65.

15. Baiardini I, Sorino C, Di Marco F, et al. Smoking cessation, anxiety, mood and quality of life: reassuring evidences. Minerva Med 2014:105(Suppl. 1 to No. 5):15-21.

16. Taylor G, McNeill A, Girling A, et al. Change in mental health after smoking cessation: systematic review and meta-analysis. $B M J$ 2014;348:g1151.

17. Chang FC, Hu TW, Lin M, et al. Effects of financing smoking cessation outpatient services in Taiwan. Tob Control 2008;17:183-9.

18. Chang FC, Hu TW, Lo SY, et al. Quit smoking advice from health professionals in Taiwan: the role of funding policy and smoker socioeconomic status. Tob Control 2010;19:44-9.

19. Hsueh $\mathrm{KC}$, Chen $\mathrm{CY}$, Yang YH, et al. Smoking cessation program in outpatient clinics of Family Medicine Department in Taiwan: a longitudinal evaluation. Eval Health Prof 2010:33:12-25.

20. Chen PC, Lee YC, Tsai ST, et al. A cost-benefit analysis of the outpatient smoking cessation services in Taiwan from a societal viewpoint. Nicotine Tob Res 2012;14:522-30.

21. EuroQol Group. About EQ-5D. Secondary About EQ-5D 1987. http:// www.euroqol.org/

22. Rabin R, de Charro F. EQ-5D: a measure of health status from the EuroQol Group. Ann Med 2001;33:337-43.

23. Naranjo A, Bilbao A, Erausquin C, et al. Results of a specific smoking cessation program for patients with arthritis in a rheumatology clinic. Rheumatol Int 2014;34:93-9.

24. Whynes DK. TOMBOLA Group. Correspondence between EQ-5D health state classifications and EQ VAS scores. Health Qual Life Outcomes 2008;6:94.

25. Whynes DK. Does the correspondence between EQ-5D health state description and VAS score vary by medical condition? Health Qual Life Outcomes 2013;11:155. 